\title{
Rotational Braking, Angular Momentum Transport and the Light Element Abundances
}

\author{
Suchitra C. Balachandran \\ Department of Astronomy, University of Maryland, College Park MD \\ 20740, U.S.A.
}

\begin{abstract}
Three critical tests are applied to the models that hypothesize the transport of angular momentum and material via turbulence. The models fail on all three counts.
\end{abstract}

\section{Introduction}

In the last decade, the model most often cited and tested for the mixing and subsequent depletion of lithium below the stellar convective zone is that which transports angular momentum and material via turbulence. Commonly referred to as the Yale rotational models (Pinsonneault et al. 1989 (P89); 1990), these models have also been by studied by Charbonnel et al. (1992; 1994). The basic premise of the models is that once the outer layers of the young star are spun-down on the main sequence, a state of differential rotation with radius is set up enabling the transport of both angular momentum and material via turbulence resulting from the shear forces between the layers. The predictions include a slow spin-down of the star, accompanied by a slow mixing process which is able to destroy surface lithium and beryllium. The models also predict that a spread in lithium will develop in a group of stars which initially have the same lithium abundance but a spread in angular momenta. Observations have essentially sought to test the latter prediction, but the existence of a spread in lithium in older clusters is not necessarily a vindication of the models. Here I will briefly describe three pieces of observational evidence that disagree with the model predictions.

\subsection{Helioseismology}

The turbulence models (P89) predict that the present-day Sun should have a rapidly rotating core. Interpretation of helioseismological data (Tomczyk et al. 1995; Charbonneau et al. 1998) indicates that the Sun is rotating slowly down to $r / R_{\odot}=0.2$. The conclusion is that the transport of angular momentum in the Sun (and by inference in other stars) is more efficient than can be achieved by turbulence, at least as modeled by P89. Alternative, more efficient models, of angular momentum transport by magnetic fields (Charbonneau \& MacGregor 1993) or by internal gravity waves (Zahn, Talon \& Matias 1997) which produce slow, rigid rotation far more rapidly may be viable alternatives. 


\section{2. $\quad$ Mixing}

The turbulence models predict the existence of deep mixing currents. Constraining the mixing in the models by the present-day solar lithium abundance, they predict the depletion of beryllium in the Sun by a factor of three (P89). Balachandran \& Bell (1998, BB98) have argued that when the continuous opacity in the UV is fully accounted for, the beryllium abundance in the Sun equals the meteoritic value. They pointed out that the inclusion of insufficient UV opacity by previous studies (King et al. 1997; Primas et al. 1997) led to the erroneous conclusion that the Sun has depleted beryllium. Additional evidence for the validity of BB98's claims is presented by Bell et al. (2000) where it is shown that when new estimates of the Fe I bound-free opacity are included, a better fit to the solar UV flux is obtained. While helioseismology permits a look at the interior of the present-day Sun, the lack of Be depletion in the Sun implies that mixing to the depth required to deplete Be has not occurred over the entire history of the Sun.

\subsection{Tidally-Locked Binaries}

Because the depletion of lithium is linked to the rotational braking of the stars, the turbulence models predict that tidally-locked binaries, especially those with very short periods, will have larger lithium abundances than single stars of the same age. Two of the best candidates which should reflect this difference are the binaries $\mathrm{BD}+22669$ and $\mathrm{BD}+23635$ in the Hyades cluster. Thorburn et al. (1993, T93) argued that these two binaries with $T_{\text {eff }}=4850$ and $4750 \mathrm{~K}$ respectively, had $\mathrm{Li}$ abundances of $\log \epsilon(\mathrm{Li})=+0.90$ and +0.69 respectively. While the Li I feature has not been detected in single stars in the Hyades at these temperatures, the $\mathrm{Li}$ abundance at $5000 \mathrm{~K}$ is near $\log \epsilon(\mathrm{Li})=0.0$, and extrapolation to cooler temperatures suggests overabundance in the binaries. However, T93's measurements were based on equivalent widths corrected for the contribution of the secondary. Given the difficulty of interpreting the line strengths from double-lined spectra, spectral synthesis is crucial for an accurate measurement.

We obtained spectra of BD+22 669 and BD+23 635 with the coudé echelle spectrograph at the $2.7 \mathrm{~m}$ telescope at McDonald observatory. Our analysis was carried out as follows. Synthetic spectra were generated for each component spectrum using the temperatures as deconvolved by Barrado y Navascues \& Stauffer (1996), standard Kurucz non-overshoot model atmospheres and the synthesis program MOOG. The temperature of the primary agrees well with T93's adopted values. The spectra were broadened to the rotational velocities of the components as listed in Griffin \& Gunn (1981) and Griffin et al. (1982). The synthetic spectrum of the secondary was Doppler shifted to the observed value and the primary and secondary spectra were added in the ratio of their observed fluxes. The final adopted flux ratios of 2.5 and 5.5 for BD+22 669 and $\mathrm{BD}+23635$ respectively are in good agreement with the $\mathrm{V}$ magnitudes of the stars and the fluxes predicted by the Kurucz models at $6700 \AA$.

Due to space constraints a figure could not be included in this text, but more details will be supplied in a forthcoming paper. The syntheses were calculated using the canonical Hyades $\mathrm{Fe}$ abundance of $[\mathrm{Fe} / \mathrm{H}]=+0.10$. We obtained a good fit to the Fe I lines in the vicinity of the $\mathrm{Li}$ I feature confirming the $T_{\text {eff }}$ and flux ratio estimates. With this analysis, we obtain $\log \epsilon(\mathrm{Li})<+0.5$ and $<-0.1$ for 
$\mathrm{BD}+22669$ and $\mathrm{BD}+23635$. Our estimates are upper limits (not detections) and the abundance levels are far lower than T93. The final abundances are entirely compatible with the single-star $\mathrm{Li}$ abundance sequence; the binaries do not exhibit an overabundance.

\section{Conclusion}

The turbulence-based angular momentum transport and mixing models fail on three predictions: rapid rotation in the solar core, deep mixing that causes $\mathrm{Be}$ depletion, in the Sun, and an increased $\mathrm{Li}$ abundance in tidally-locked binaries. Angular momentum transport must proceed by more efficient processes, perhaps magnetic fields or internal gravity waves. Solar $\mathrm{Li}$ depletion is probably related to localized mixing at the tachocline. The vast plethora of $\mathrm{Li}$ abundances in open clusters remains to be understood in these terms.

Acknowledgments. The author is pleased to acknowledge support from NSF grant AST-9819870. Travel to the IAU General Assembly was funded by grants from the IAU, NSF and NASA.

\section{References}

Balachandran, S. C. \& Bell, R. A 1998, Nature, 392, 791 (BB98)

Barrado y Navascues, D. \& Stauffer, J. R. 1996, A\&A, 310, 879

Bell, R. A., Balachandran, S. C., \& Bautista, M. 2000, ApJ, in press

Charbonneau, P. \& MacGregor, K. B. 1993, ApJ, 417, 762

Charbonneau, P., Tomczyk, S., Schou, J., \& Thompson, M. J. 1998, ApJ, 496, 1015

Charbonnel, C., Vauclair, S., \& Zahn, J.-P. 1992, A\&A, 255, 191

Charbonnel, C., Vauclair, S., Maeder, A., Meynet, G., \& Schaller, G. 1994, A\&A, 283, 155

Griffin, R. F. \& Gunn, J. E. 1981, AJ, 86, 588

Griffin, R. F., Mayor, M., \& Gunn, J. E. 1982, A\&A, 106, 221

King, J. R., Deliyannis, C. P., \& Boesgaard, A. M. 1997, ApJ, 478, 778

Pinsonneault, M. H., Kawaler, Sofia, S., \& Demarque, P. 1989, ApJ, 338, 424

Pinsonneault, M. H., Kawaler, S. D., \& Demarque, P. 1990, ApJS, 74, 501

Primas, F., Duncan, D. K., Pinsonneault, M. H., Deliyannis, C. P., \& Thorburn, J. A. 1997, ApJ, 480, 781

Thorburn, J. A., Hobbs, L. M., Deliyannis, C. P., \& Pinsonneault, M. H. 1993, ApJ, 415, 150 (T93)

Tomczyk, S., Schou, J., \& Thompson, M. J. 1995, ApJ, 448, L57

Zahn, J.-P., Talon, S. \& Matias, J. 1997, A\&A, 322, 320 